\title{
L'écoute (L'Eglise-famille de Dieu, lieu de l'écoute de la Parole de Dieu)*
}

\author{
Roger Houngbedji D.**
}

Recibido: 3 de junio de 2014 • Aprobado: 4 de agosto de 2014

\section{Résumé}

Ecouter, du point de vue biblique, c'est s'engager existentiellement à mettre en pratique la Parole de Dieu au point qu'elle s'incarne dans la vie du croyant. L'écoute dans cette perspective est à la fois obéissante et confiante en ce sens qu'elle est détachée de toute idée de contrainte et de soumission aveugle. Cette compréhension de l'écoute trouve une résonnance particulière chez les Pères de l'Eglise qui, dans une perspective plutôt pastorale, invitent à une lecture spirituelle de la Parole de Dieu dans le but d'en être transformé. Dans l'optique d'une réflexion sur l'inculturation, l'écoute comprise comme une attitude obéissante et confiante est une donnée fondamentale pour l'émergence de l'Eglise-famille de Dieu en Afrique. L'avènement effectif de cette dernière exige que les membres de la nouvelle famille de Dieu s'inscrivent dans une démarche d'écoute de type kénotique et extatique.

Mots-clés: Écouter, Parole de Dieu, Église-Famille de Dieu, obéissance, confiance.

* Este artículo es resultado de investigación y estudio especializado del autor.

* Dominicain, de nationalité béninoise, docteur en Ecriture Sainte, il est professeur à l'Université Catholique de l'Afrique de l'Ouest/Unité Universitaire d'Abidjan (UCAO/UUA) et au Centre Dominicain de Théologie et de Développement de Yamoussoukro. Il a publié plusieurs articles et ouvrages dont les plus récents sont: L'Eglise-famille de Dieu en Afrique, selon Luc 8, 18-21. Problèmes de fondements, publié aux Ed. UCAO (2008) et L'Harmattan (2009); La radicalité de la vie religieuse en Afrique, publié aux Ed. Paulines (2010).

Résidence: Fraternité Saint Dominique d'Abidjan, 08 BP 2675 Abidjan 08 (Côte d'Ivoire). Tél. (225) 081211 33. E-mail: houngber@hotmail.com 


\title{
Listening (The Church-Family of God, a listening place for the Word of God)
}

\begin{abstract}
Hearing from the biblical point of view is an existential commitment to put in practice the Word of God, so as it could be rooted in the faithful life. In this line, hearing should be understood as obedience and confidence in the sense that it does not admit any idea of submission and constraint. This understanding is echoed by the early Fathers of the Church in a pastoral point of view as an invitation to a spiritual reading of the Word of God in the view of being transformed. In the line of a theological reflection on inculturation, hearing as obedience and confidence is fundamental for the emerging of the Church as Family of God in Africa. The effective setting-up of such a Church requires from the members of the new Family of God a kenotic and ecstatic hearing.
\end{abstract}

Keywords: Listen, Word of God, Church-Family of God, obedience, confidence.

\section{Escuchar (La Iglesia-Familia de Dios, lugar de escucha de la palabra de Dios)}

\section{Resumen}

Escuchar, desde la óptica bíblica, es comprometerse existencialmente a aplicar la Palabra de Dios hasta el punto de incorporarla en la vida del creyente. Desde esta perspectiva, La Escucha (de la palabra de Dios) es dócil y confía en el sentido en el que está completamente separada de cualquier idea de limitación y sumisión ciegas. Desde Los Padres de la Iglesia se hace una reflexión particular sobre este entendimiento de la escucha, quienes desde una perspectiva principalmente pastoral, invitan a realizar una lectura espiritual de la Palabra de Dios, con el fin de ser transformados por ella. En el contexto de reflexión sobre la inculturación, la escucha entendida como una actitud de obediencia y confianza, es un elemento primordial en el surgimiento de la Iglesia - Familia de Dios en África. Esto requiere que los miembros de la Nueva Familia de Dios formen parte de una dinámica de escucha de tipo kenótica y extática.

Palabras clave: escucha, Palabra de Dios, Iglesia-familia de Dios, obediencia, confianza. 


\section{Introducción}

Le thème de l'écoute est de nos jours un des champs explorés par les théologiens de l'Eglise-famille de Dieu en Afrique ${ }^{1}$. Pour ces derniers en effet, l'écoute de la parole apparaît comme une des conditions pour qu'advienne l'Eglise-famille de Dieu en Afrique. Le thème de l'écoute a donc toute son importance pour la réflexion théologique en contexte africain. Dans cet article nous nous proposons dans un premier temps de cerner le sens de l'écoute en milieu africain, notamment à partir de l'aire culturelle adja-fon du Bénin. Cette étude lexico-anthropologique nous permettra ensuite d'explorer tous les aspects de sens que recouvre ce thème à partir de son enracinement biblique et en rapport avec les commentaires des Pères de l'Eglise. Enfin, nous nous interrogerons sur ce qui fait la spécificité de l'écoute biblique par rapport à l'écoute en contexte africain.

\section{Le sens de l'écoute dans l'aire culturelle Adja-fon du Bénin}

L'Afrique étant plurielle et vu les limites imposées à cet article, nous proposons de centrer notre étude sur l'aire culturelle Adja-fon du Bénin où la question de l'écoute en rapport avec le thème de la famille est vivement posée. En effet, dans la vision de l'homme fon, l'expression typique pour parler de l'écoute est: se / to / $n u$, ce qui se traduit littéralement par écoute / oreille / chose, c'est-à-dire: "l'écoute de la chose faite pour l'oreille". La chose dont il s'agit ici est la parole qui en fon se traduit par $x o$. Il s'établit ainsi un rapport étroit entre l'écoute, comprise comme obéissance, et la parole. L'oreille, par sa forme symbolique, se présente ici comme le réceptacle de la parole, le lieu de «l'accueil de ce qui est réellement facteur de vie et de cohésion sociale» ${ }^{2}$. Dans ce sens, pour intimer l'ordre à quelqu'un d'écouter, les Adja-fons diront yi / se (accueille / écoute!), d'où vient l'expression:

1 Cf. R. Houngbedji, L'Eglise-famille de Dieu en Afrique selon Luc 8, 19-21. Problèmes de fondements, Paris, L'Harmattan, 2009.

2 Nathanaël Y.Soede, «Parole et vérité dans la culture et les comportements actuels des africains», in RUCAO 24 (2005), p. 146. Voir aussi dans le même sens D. Zahan, La dialectique du verbe chez les Bambara, Paris, Mouton \& Co, 1963, p. 24. L'auteur ici fait remarquer le caractère inséparable de la parole et de l'oreille chez les Bambara. Pour ceux-ci en effet sans audition, il n'y a pas de parole possible. 
yi / se / non / lè (ceux qui accueillent et écoutent), c'est-à-dire les croyants. Ecouter dans cette perspective, c'est donc obéir, prêter l'oreille à la parole qui s'énonce.

Pour l'homme fon, le premier lieu où la parole doit être écoutée est le cadre de l'éducation. Celle-ci commence par l'attention à porter au réel. En effet, tout réel est porteur d'un nom (nu-nyi) au travers duquel une parole $\left(x_{0}\right)$ se donne à entendre. En portant l'attention au réel, on veut donc surtout accéder à la parole (xo) qu'il sous-tend et qui se donne à entendre. En milieu fon, le sage (l'ancien) est celui qui d'expérience connait les noms des choses au point de devenir le $m \hat{e}$ $x o$-qui littéralement signifie "personne-parole"-, c'est-à-dire celui qui le premier a écouté la parole et s'est identifié à la personne qui la prononce. Le respect dû à sa personne s'explique par le fait que la longue expérience de la vie lui a permis d'être suffisamment à l'écoute du xo pour être capable de gérer son savoir des choses. L'homme ne peut en effet avoir un rapport aux réalités de la vie et les nommer dûment que dans une attitude d'écoute qui le maintienne ouvert au sens invisible des choses, c'est-à-dire à la parole originelle venant d'ailleurs.

De fait, la parole a sa source première dans l'Etre suprême, le créateur du monde et de la vie (gbêdoto). C'est parce qu'elle provient d'au-delà du monde de l'homme qu'elle est source de vie $\left(x_{0} \text {-joxo }\right)^{3}$, génératrice de la vie familiale et communautaire. Cependant l'écoute de la parole provenant du créateur suppose la médiation des ancêtres, appelés mêxoxo (c'est-à-dire l'Ancien des jours ${ }^{4}$ ), et des aînés (mêxo), lesquels sont dépositaires de la parole du fait de s'y être identifiés, et sont chargés de la transmettre dans une tradition (hwendo) vivante. Du coup, «écouter la parole en milieu fon, c'est actualiser la tradition (huendo), la rendre vivante. C'est en effet au cour de la tradition que la parole originelle $\left(x_{0}\right)$ livrée par le premier ancêtre prend corps et se communique aux générations postérieures. Celles-ci sont appelées à écouter la parole inscrite dans la tradition tout en gardant un rapport d'ouverture vers la source. C'est dans ce processus d'intériorisation de la tradition (lieu de l'écoute du $x o$ ) que s'effectue l'intégration de l'individu dans sa famille et que celle-ci se constitue» ${ }^{5}$. Il y a lieu de voir les soubassements bibliques de cette écoute, telle que définie en contexte africain.

3 L'expression xo-joxo en fon veut dire littéralement "parole-engendrant parole", c'està-dire parole féconde, fertile, susceptible de donner la vie. Voir dans ce sens J. M. Agossou, Christianisme africain. Une fraternité au-delà de l'ethnie, Paris, Karthala, 1987, p. 69. 71. 100.

4 Cf. B. Adoukonou, Jalons pour une théologie africaine. Essai d'une herméneutique chrétienne du Vodoun dahoméen, T2, Etude ethnologique, Lethielleux, Paris, 1980, p. 135.

5 R. Houngbedji, L'Eglise-famille de Dieu en Afrique..., p. 257-258. 


\section{Enracinement biblique du terme écoute}

L'écoute apparaît comme un des thèmes majeurs de la Bible qui traduit le rapport de l'homme à Dieu et à sa parole. Le Dieu de la Bible se présente en effet comme le Dieu de la parole, et c'est parce qu'il parle que l'homme, un être historique et parlant, peut répondre à travers une vie de foi qui naît de l'audition de la parole (cf. Rm 10,14). Ainsi toute l'histoire du salut se présente comme l'histoire de la révélation de la Parole de Dieu, l'auto-communication de Dieu à son peuple, dont la manifestation définitive s'accomplit en son Fils Jésus-Christ, le Verbe de Dieu incarné dans notre histoire (cf. He 1, 1-4). Toute la question est de savoir comment accéder à la parole divine. Autrement dit, dans quelle disposition l'homme peut-il accueillir la Parole de Dieu à lui adressée, de sorte qu'elle réalise dans sa vie la mission qui lui est assignée (cf. Is 55, 11), c'est-à-dire l'œuvre de la régénérescence et de la rédemption?

\subsection{L'écoute dans la bible hébraïque}

Dans la bible hébraïque le mot "écoute" est exprimé par la racine עמש (shema) dont le verbe correspondant shama signifie, selon le contexte, "entendre", "écouter", "obéir", voire "apprendre". L'expression biblique "shema Israël", bien connue dans les Saintes Ecritures et très caractéristique du peuple de la bible, veut souligner ce type d'écoute qui a marqué le peuple d'Israël tout au long de son histoire et dans son rapport particulier à Dieu. Une autre racine hébraïque $\mathrm{x}$, très liée à la première, est connue pour le substantif "oreille" et comme verbe "prêter l'oreille ou "tendre l'oreille". Ce dernier vocable sert souvent de parallèle synonymique à עמש lequel demeure le terme le plus important dans la bible pour exprimer l'attitude d'écoute.

A travers l'expression typique "Ecoute, Israël", le peuple de la bible est donc invité à écouter, à prêter l'oreille ou à obéir. La question qui, d'entrée de jeu, mérite d'être posée est celle de savoir l'objet de cette écoute: qu'est-ce que le peuple d'Israël doit écouter ou à qui il doit prêter l'oreille, obéir? Comment peut-il assumer cette qualité d'écoute pour mieux correspondre aux attentes de Dieu? Pour mieux cerner tous les aspects de sens liés à cette expression, il importe de la situer dans le contexte deutéronomique où elle apparaît et semble très usitée.

L'expression biblique "Ecoute Israël" (Dt 6,4) se situe dans un grand ensemble qui va du chapitre 6,4 au chapitre 9,29 du livre de Deutéronome. Elle forme une inclusion introduisant le début de chacune des deux sections, ce qui 
en souligne bien l'importance. La séquence où est insérée l'expression est immédiatement précédée par la section des Dix Commandements (Dt 5,23-6,3) et elle s'inscrit dans le mouvement d'ensemble des chapitres 5 à 11 qui traitent du récit fondateur du don des Dix Paroles au peuple de Dieu. Moïse, après avoir attiré l'attention du peuple sur le don des Dix Paroles que Dieu lui fait, l'invite à l'écoute de ces paroles par l'injonction: "Ecoute, Israël". En quoi consiste cette écoute et que vise-t-elle?

L'écoute inscrite dans l'injonction adressée par Moïse au peuple d'Israël invite d'abord à une confession de foi -"Yahvé notre Dieu est Yahvé unique"-, laquelle est un acte de reconnaissance affirmant que celui qui porte le nom de Yahvé est unique. Unique doit être saisi ici non seulement dans le sens de "l'unicité de Dieu"6 mais aussi comme "l'acte par lequel quelqu'un dit d'un autre qu'il est unique à ses yeux"7. Autrement dit, c'est parce que Yahvé est unique aux yeux d'Israël, comme dans une relation d'amour, qu'il le reconnait comme son Dieu. Et de fait, la confession de foi, lieu de reconnaissance, est immédiatement suivie par le commandement de l'amour: «Tu aimeras le Seigneur ton Dieu de tout ton cœur, de tout ton être, de toute ta force...» (Dt 6, 5-9).

L'écoute biblique est donc étroitement liée à l'amour: le Dieu dont il faut écouter la Parole est celui qui a pris l'initiative d'aimer Israël et ce dernier le reconnait et le confesse comme tel. En effet, «comment écouter quelqu'un, croire en lui et lui faire confiance si nous n'avons pas l'assurance que cette personne nous aime et veut par-dessus tout notre bien?» ${ }^{8}$. Or comment se concrétise l'amour de Dieu vis-à-vis de son peuple? L'amour de Dieu pour Israël se concrétise par le don de ses Paroles (Devarim), c'est-à-dire le Décalogue ou la Torah (Loi). Cette Loi se présente comme un chemin révélé par Dieu pour conduire l'homme à la vie. La Loi, don précieux de Dieu pour l'homme, est donc d'abord et avant tout une source de vie et de bonheur. C'est ce bonheur que reconnait le psalmiste en exaltant les bienfaits de la Loi ${ }^{9}$. L'écoute biblique se présente donc comme un

6 Cf. D. L. Christensen, World Biblical Commentary Vol. 6A. Deuteronomy I-II, Dallas, Word Books, Publisher, 1991, p.145.

7 J. M. Carriere, Le livre du Deutéronome. Choisir la vie, Paris, Editions Ouvrières, 2002, p.176.

8 M. Hubaut, Un Dieu qui parle! Comment Dieu se révèle-t-il à l'homme?, Paris, Cerf, 2012, p. 119.

9 Voir à ce propos les psaumes 19 et 119. 
engagement à marcher sur le chemin, les Paroles révélées par Dieu dans la Torah, pour parvenir à la vraie vie.

Il s'agit là d'une question de vie ou de mort, de malheur ou de bonheur. D'où l'importance pour l'homme de s'engager à suivre ce chemin de vie de tout son cœur. Le cœur, au sens biblique du terme, est en effet le lieu privilégié, le plus intime de mon être «où je dois accueillir, ruminer, méditer cette Parole de vie pour la mettre en pratique» ${ }^{10}$. Lorsqu'Israël est donc convié à l'écoute, ce qu'on attend de lui, c'est l'engagement existentiel de toute sa personne vis-à-vis de la Loi de Dieu: sa vie entière doit être polarisée par la mise en pratique de cette Loi, dans la mesure où elle conduit à la vraie vie. Dans la mise en pratique de la Loi, est exclue toute idée de contrainte ou de soumission aveugle. Ce qui est en jeu ici est plutôt de l'ordre de la libre adhésion du cœur, un acte de foi et d'amour. De ce point de vue, l'écoute biblique est «synonyme de la foi de l'homme qui fait confiance à Dieu, convaincu qu'il sait, lui, le Créateur, ce qui peut construire ou détruire l'homme. Elle n'est pas une soumission d'esclave mais une démarche d'homme libre enraciné dans l'amour du Créateur» ${ }^{11}$.

Parler de la démarche d'homme libre, c'est toucher au point critique où se joue pour le sujet de l'écoute la question de la vie ou de la mort. L'homme est en effet libre de suivre ou non le chemin de vie que la Parole de Dieu lui révèle. A cause du péché, il a tendance à vouloir faire sa vie tout seul, loin de Dieu, attitude qui correspond à un refus d'écouter la Parole (la Torah) de Dieu. La seule condition pour l'homme pécheur de retrouver le chemin de la vraie vie et du bonheur est la conversion -terme qui vient de l'hébreu shûb-qui étymologiquement veut dire "retour" vers Dieu. Après s'être éloigné de Dieu, l'homme pécheur est donc appelé à rebrousser chemin, à revenir au Dieu de l'Alliance. C'est ici que prennent sens toutes les vives interpellations des prophètes invitant Israël à revenir au Seigneur (Cf. Os 6,1-6; Is 1, 11-17; Jr 3, 12-13; 18,12; 31,18-19; Ez 18, 31-32; 33, 10-11; 36, 25-28). Dans cette perspective, écouter la Parole de Dieu, c'est aussi se convertir, détourner son cœur du chemin des idoles et de la mort pour le "retourner" vers Dieu. On parlera dans ce sens de la "circoncision du cœur", expression biblique qui indique que le cœur, centre de la personne, est le lieu qui doit subir le véritable retournement pour que s'effectue une écoute profonde de la Parole.

10 M. Hubaut, Op. Cit., p. 123.

11 M. Hubaut, Op. Cit., p. 121. 
Ecouter dans la perspective de la religion d'Israël, ce n'est donc pas prendre connaissance intellectuellement de la Parole de Dieu ou d'une information sur Dieu, mais s'engager plutôt de façon existentielle à vivre la Parole de Dieu, à marcher sur le chemin qu'elle trace, lequel chemin conduit au bonheur, à la vraie vie. L'écoute ici est d'abord et avant tout une écoute-obéissante qui permet à l'homme de vivre une intimité profonde avec Dieu, source de la vraie vie. Cette dimension de l'écoute semble ce qui apparaît le plus dans les textes néotestamentaires.

\subsection{L'écoute dans les textes néotestamentaires}

Le verbe "écouter" dans le Nouveau Testament est la traduction du mot grec akouô qui a plusieurs variantes. L'une de ces variantes est le terme hupakouô (composé de hupo + akouô) qui veut dire "obéir à", avec l'idée de soumission ${ }^{12}$. Nous retrouvons ici l'idée de l'écoute-obéissante, telle que définie dans le premier testament. Quels sont les points caractéristiques de cette écoute dans la perspective des textes néotestamentaires?

Un des points dominants mis en exergue par les textes du Nouveau Testament est le rapport étroit entre l'écoute et la mise en pratique de la Parole de Dieu. Ce rapport apparaît dans plusieurs passages de l'Ecriture, notamment chez Luc, auteur du troisième évangile et des Actes des Apôtres. Dans la perspective lucanienne, l'écoute de la Parole de Dieu est intrinsèquement liée à sa mise en pratique: on ne peut imaginer un "écoutant" de la parole qui ne soit en même temps un "pratiquant", on n'est qualifié de sujet d'écoute que dans la mesure où on le démontre dans les faits. La parole réellement écoutée doit se voir dans les actes concrets, fruits de la parole fécondante.

L'épisode des frères et sœurs de Jésus rapporté en Lc 8, 19-21 fait bien apparaître ce lien. Dans ce texte, Luc met en rapport les deux verbes principaux par lesquels il exprime l'écoute et la mise en pratique de la Parole de Dieu: "akouontes kai poiountes". La préposition "kai" (et) qui lie les deux verbes mis au participe présent montre bien qu'on ne peut séparer "l'écoute" du "faire", les deux actions étant intimement liées: l'écoute exige le faire et le faire, l'écoute. Par ailleurs, du point de vue grammatical, la forme du participe présent manifeste un état duratif de l'action: l'écoute et le faire ne sont pas des actions ponctuelles, elles doivent durer dans le temps. Autrement dit, c'est dans la mesure où nous demeurons dans l'écoute et la mise en pratique de la parole que nous pouvons prétendre

12 Cf. E. Käsemann, Essais exégétiques, Neuchâtel, Delachaux \& Niestlé, 1972, p. 93. 
être de la famille de Jésus. Un autre élément souligné fortement par Luc dans cet épisode est le lien avec la parabole du semeur (Lc 8, 4-18) qui précède immédiatement la péricope susmentionnée. La parabole du semeur est en effet le lieu où Luc souligne clairement le lien entre l'écoute et la mise en pratique de la Parole de Dieu. Il y montre en effet comment la parole bien écoutée ${ }^{13}$ donne lieu à une fructification, une production du fruit au centuple (Luc 8, 8. 15). La non-production sera alors vue comme le signe d'un manque d'accueil et d'écoute de la parole.

Cette compréhension de l'écoute se répercute dans d'autres passages de l'Ecriture (cf. Lc 11, 28; 6, 46-48; Mt 12, 46-50; 21, 28-31; Mc 3, 31-35) où les auteurs soulignent fortement le lien indissociable entre la parole écoutée et sa mise en pratique, ce qui correspond aussi à l'accomplissement de la volonté de Dieu (cf. Mt 12, 50; Mc 3, 35). Autrement dit, le disciple de l'écoute ne doit pas se contenter d'écouter superficiellement la parole mais l'incarner dans un vécu qui rend visible et atteste que la parole de Dieu accueillie (lieu de la manifestation de la volonté de Dieu) porte du fruit dans sa vie. Nous sommes ici en plein cœur du mystère de l'Incarnation: écouter la Parole de Dieu, c'est lui donner corps dans le vécu quotidien, c'est rendre visible le Verbe de Dieu qui se rend présent à travers la vie concrète et fécondée du disciple.

Un autre aspect de l'écoute qui ressort des textes néotestamentaires est son rapport à la foi: l'écoute biblique suppose la foi, sans laquelle elle ne peut porter de fruit. L'évangéliste saint Jean est celui qui souligne fortement cet aspect de l'écoute: «Celui qui écoute ma parole et croit à celui qui m'a envoyé a la vie éternelle et ne vient pas en jugement mais il est passé de la mort à la vie» $(J n 5,24)$. La foi ici est l'ouverture à Dieu, la vie d'intimité avec Dieu, qui seul peut nous révéler le sens profond de sa parole et mettre en nous les dispositions intérieures nous permettant d'entendre sa voix (Jn 5, 25; 18, 37) et d'accueillir positivement sa Parole (cf. Jn 6, 44-45). L'apôtre Paul, dans le même sens, parlera de l'obéissance de la foi (akoês pisteôs: Ga 3, 2. 5), obéissance qui loin d'être une soumission aveugle ou passive se présente plutôt comme une écoute du cœur disposant à accueillir l'Evangile (Rm 10, 14-17).

C'est dire que dans la perspective biblique, il y a une identité entre l'écoute et la foi comme acceptation confiante de la Parole de Dieu, obéissance totale à la volonté de Dieu. Dans la perspective de l'œuvre de la Rédemption accomplie par le Christ, ce dernier est celui en qui s'accomplit éminemment l'écoute obéissante

13 La récurrence du terme " écouter » dans cette section (Lc 8, 8. 10. 13. 14. 15) montre bien son importance chez Luc et son rapport avec l'idée de la fructification ou production. 
de la foi, en ce sens que par son obéissance et sa soumission totale à Dieu son Père, il s'ouvre entièrement à la Parole l'invitant à l'accomplissement du salut du genre humain. Du coup, se dessaisissant de sa vie et «devenant obéissant jusqu'à la mort, et la mort sur une croix» (Ph 2, 8), il conduit l'homme à la vraie vie et au bonheur. L'acte d'abaissement et de soumission du Christ se présente ainsi comme le lieu où s'effectue un décentrement total de lui-même (sa kénose) pour apporter la vie à l'humanité.

L'écoute, du point de vue néotestamentaire, apparaît donc comme le lieu où en Christ (Verbe de Dieu incarné) se réalise le mystère pascal: passage de la mort à la vie, passage de l'esclavage à la liberté. Le disciple du Christ qui aspire à cette liberté et à cette vie est irrémédiablement appelé à s'inscrire dans cette dynamique de l'écoute pascale qui lui permet d'effectuer un total décentrement de lui-même pour être entièrement donné au Christ et au prochain. Il y a lieu de se demander la résonnance que trouve cette compréhension de l'écoute biblique chez les Pères de l'Eglise.

\subsection{L'écoute chez les Pères de l'Eglise}

Le thème de l'écoute trouve une résonnance particulière chez les Pères des premiers siècles de l'Eglise, notamment dans le sens de la Lectio Divina, entendue comme lecture spirituelle de la Parole de Dieu dans le but de s'en nourrir et d'en être transformé. Le but visé dans cette pratique est la rencontre de Dieu dans la parole écoutée, d'où l'invitation à une écoute effective et transformatrice. De ce point de vue, les commentaires de certains Pères méritent une attention particulière.

Pour Saint Césaire d'Arles ${ }^{14}$, les Saintes Ecritures sont comme «des lettres venues de notre patrie» (paradis), par lesquelles nous sommes invités dans notre «éternelle et première patrie». Autrement dit, les Saintes Ecritures que les chrétiens sont appelés à lire, à scruter pour s'en nourrir, sont effectivement la Parole de Dieu, transmise par " les patriarches, les prophètes, les apôtres et les martyrs", les instruisant sur les réalités célestes, notre éternelle et première patrie. Le chrétien situé dans ce monde est en effet un pèlerin, il est en marche vers le Royaume de Dieu, la patrie céleste. D'où la nécessité pour lui d'accueillir les Saintes Ecritures comme une Parole venant de Dieu et le préparant spirituellement à entrer dans son Royaume. Dans ce sens, le chrétien doit éviter le péché d'orgueil,

14 Saint Cesaire D'Arles,, Sermons au peuple, Tome I (Sermons 1-20), Paris, Cerf, 1971. 
présenté ici comme un esprit de rébellion aux Saintes Ecritures. Si le Christ s'est incarné, c'est justement pour nous libérer du péché, ou plus précisément, de la tyrannie et de l'orgueil du diable. Il y a donc lieu de veiller à toujours lutter contre l'esprit de rébellion qui en nous peut empêcher d'être à l'écoute de la Parole de Dieu, source de notre salut.

Par ailleurs, la Lectio Divina exige une persévérance, un effort continuel à fournir dans la lecture et l'écoute des textes. L'image du cultivateur est utilisée ici par l'auteur pour mieux souligner cette idée d'effort et de persévérance. En effet, «les cultivateurs s'efforcent de semer différentes sortes de semences afin de pouvoir se préparer une nourriture suffisante pour eux-mêmes et pour les leurs. Combien plus, lorsqu'il s'agit de bénéfices spirituels, ne devez-vous pas vous contenter d'entendre lire la Parole de Dieu à l'église : vous devez prolonger la lecture sacrée dans vos maisons, au cours de vos repas, et, quand les jours sont courts, y consacrer encore quelques heures de nuit. C'est ainsi que vous amasserez un froment spirituel dans le grenier de votre cœur et rangerez dans le trésor de vos âmes les perles précieuses des Écritures.» ${ }^{15}$

Cette lecture et écoute persévérante de la Parole de Dieu est d'autant plus importante que personne ne peut s'en excuser, car il y va de la vie de l'âme dans l'éternité et du salut de l'homme. De ce point de vue, l'exhortation de Saint Césaire se fait plus pressante: «Je vous prie, frères bien-aimés, de vous appliquer à consacrer à la lecture des textes sacrés autant d'heures que vous le pourrez. Et puisque les lectures... dont nous avons voulu nous munir dans cette vie sont la nourriture de l'âme pour l'éternité, que personne ne cherche d'excuse en disant qu'il n'a pas du tout appris à lire ; car ceux qui ne savent pas lire, s'ils aiment vraiment Dieu, essaient de trouver des gens instruits capables de leur lire les saintes Ecritures» ${ }^{16}$.

L'écoute de la Parole de Dieu se présente donc ici comme une nourriture dont l'homme intérieur, fait à l'image et à la ressemblance de Dieu, ne peut se passer s'il veut vivre et croître en Dieu. Ce point de vue est partagé par plusieurs autres Pères de l'Eglise, tels que Guillaume de Saint-Thierry (pour qui l'obéissance très fidèle à la Parole de Dieu remplit la vie du chrétien de la "joie spirituelle") ${ }^{17}$ et Grégoire de Nysse qui montre comment "écouter" la Parole de Dieu,

15 Idem, p. 341

16 Saint Cesaire D'Arles, Op. Cit., p. 349.

17 Guillaume de Saint-Thierry, Monition sur la méditation de la Parole de Dieu (Sermon 6-9), in Sources Chrétiennes, 175, Paris, Cerf, 1971. 
c'est "boire" à une source intarissable, permettre au "Verbe une entrée dans son âme", être "dépouillé du vieil homme" pour "revêtir la tunique nouvelle" qu'est le Christ ${ }^{18}$.

Saint Augustin, quant à lui, traitant plus explicitement du thème de l'écoute situe le débat sur un plan philosophique. En effet, pour lui, la question de l'écoute touche à ce qu'il y a de fondamental dans l'humanité de l'homme, c'est-à-dire «ce qui confère, chaque fois, à l'existence humaine sa stature» ${ }^{19}$. L'homme ne peut en effet tenir debout que parce qu'il est à l'écoute de la parole qui provoque en lui la joie de vivre, tout comme «l'ami de l'époux est ravi de joie à la voix de l'époux» $(J n 3,29)$. Dans le même temps, toute déficience dans l'écoute est cause de la chute et de l'affaissement de l'homme. L'écoute se présente donc comme quelque chose de fondamental dans la vie de l'homme appelé à se tenir toujours debout, c'est-àdire ouvert et disposé à accueillir la parole de l'Epoux qui le fait vivre.

La figure biblique choisie par saint Augustin pour caractériser ce type d'écoute est celle de Marie, assise aux pieds du Seigneur pour écouter sa parole (Luc 10, 39). Dans son commentaire sur ce passage de l'Ecriture, saint Augustin, comparant Marie à sa sœur Marthe, précise: «Marie s'abandonnait tout entière à la suavité de la parole divine. Marthe n'avait qu'un souci: comment nourrir le Seigneur; Marie n'en avait qu'un: comment être nourrie par lui. Marthe préparait un festin au Seigneur; un autre festin faisait déjà les délices de Marie» ${ }^{20}$. Ecouter, c'est donc participer au festin de la parole, une parole qui est nourriture en ce sens qu'elle «entretient notre vie et nos forces, nous donne la capacité d'agir, nous restaure en tout sens» ${ }^{21}$.

Du coup, pour saint Augustin, il existe un rapport étroit entre l'écoute, l'obéissance et la vie: «Qui écoute et obéit (qui audit et obaudit) vivra; qui écoute et n'obéit pas, c'est-à-dire qui écoute avec mépris, qui écoute sans croire, ne vivra pas. Pourquoi ne vivra-t-il pas? Parce qu'il n'écoute pas. Que signifie: il n'écoute pas? Il n'obéit pas» ${ }^{22}$. Ecouter, c'est donc obéir à la parole entendue, de sorte qu'on puisse en vivre comme une nourriture qu'on mange, qu'on digère et qu'on

18 Gregoire de Nysse, Homélie sur le Cantique des Cantiques, Paris, Migne, 1992, p. 178-207.

19 J. L. Chretien, Saint Augustin et les actes de parole, Paris, PUF, 2002, p. 25.

20 Saint Augustin, Sermo 104, 1, trad. Humeau, Les plus beaux sermons de saint Augustin, Paris, 1986, t. II, p. 148.

21 J. L. Chretien, Saint Augustin et les actes de parole... p. 31.

22 Ibidem, p. 30. 
rumine. Le lien entre l'écoute et la vie se fonde dans la solidarité de l'écoute et de l'alliance avec Dieu, c'est-à-dire la vie d'intimité avec Dieu. En effet, «Dieu ne peut être mon Dieu que là où je l'écoute. "Ecoute, mon peuple et je te parlerai" ${ }^{23}$. Dans ce sens, celui qui parle comme celui qui écoute doit être à l'écoute de Dieu qui lui parle au fond du cœur, car «on ne parle en vérité que pour autant qu'on écoute et aussi longtemps qu'on écoute» ${ }^{24}$. L'écoute exige donc ici les oreilles du cour (aures cordis), lesquelles permettent d'accueillir et de discerner la voix de Dieu qui nous parle.

Il en ressort que pour les Pères de l'Eglise, l'écoute est fondamentalement l'attitude d'obéissance et d'ouverture qui met le croyant en rapport avec la Parole de Dieu, laquelle constitue la source véritable (la vraie nourriture) à laquelle il doit se référer pour avoir la vie et la consistance. Ainsi, chez les Pères de l'Eglise comme dans les Saintes Ecritures, l'écoute est l'engagement à vivre la Parole de Dieu comme lieu de décentrement, de sorte que celle-ci s'incarne en l'homme et le conduise à la vraie vie. Dans la perspective d'une réflexion sur l'inculturation, quel rapport y a-t-il entre l'écoute biblique et l'écoute en contexte africain?

\section{Spécificité de l'écoute biblique dans son rapport à l'écoute en contexte africain}

L'écoute biblique dans son rapport à la culture africaine manifeste quelques points communs que l'étude antérieure nous permet de relever. D'abord, il est à noter que l'écoute garde un lien étroit avec la parole présentée comme une réalité transcendante, provenant d'ailleurs et susceptible de générer une nouvelle vie dans la personne qui l'accueille. Dans l'histoire de la Révélation, cette parole est celle révélée au cours des siècles par le Dieu de l'Alliance au peuple d'Israël et qui, dans les temps derniers, s'est manifestée de façon définitive en son Fils, le Verbe incarné de Dieu (cf. He, 1, 1-4). Dans cette perspective, écouter la Parole de Dieu, c'est s'engager à la mettre en pratique dans une totale obéissance à la manière du Fils de Dieu qui, devant accomplir la volonté du Père, s'est soumis à sa parole jusqu'à la mort sur la croix (Ph 2, 8). La Parole de Dieu écoutée est alors

23 Ibidem, p. 31.

24 J. L. Chretien, Op. Cit., p. 28. 
génératrice d'une nouvelle vie, la plénitude de vie à laquelle aspire le disciple du Christ (cf. Jn 10, 10).

Dans le même sens, l'écoute en contexte africain, notamment le milieu fon, met en rapport avec la parole principielle $\left(x_{0}\right)$ venant du créateur du monde (gbêdoto) et que les ancêtres (mêxoxo) et les sages (mêxo) ont le devoir de transmettre fidèlement à travers une tradition vivante (hwendo) aux membres de leur famille, de sorte que celle-ci soit revivifiée et consolidée. L'écoute ici est aussi le lieu d'une obéissance radicale à la parole fondatrice, véhiculée par la tradition et incarnée dans la personne du sage (mêxo) qu'il faut absolument écouter afin de jouir d'une vie épanouissante, susceptible de redynamiser les rapports fraternels au sein de la communauté familiale.

Cependant, si l'écoute biblique inscrite en contexte africain fait apparaître des points de convergence indéniables, les lieux de divergence semblent plus déterminants. D'un côté comme de l'autre, tout semble tourner autour de la façon dont s'effectue l'écoute obéissante. Alors que l'écoute en contexte de la Révélation est fondamentalement une obéissance-confiante où prédomine une relation d'amour avec le Dieu d'amour, en contexte africain l'écoute apparaît comme un ordre moral auquel le sujet d'écoute est contraint d'obéir, sinon il court le risque de n'être pas intégré dans la communauté.

En effet, l'écoute en milieu fon exige la mise à exécution scrupuleuse de la parole inscrite dans la tradition et que l'ancien a le devoir, voire l'obligation, de faire observer. L'écoute-obéissante ici ne laisse pas de champ libre à l'initiative personnelle ni à la manifestation d'une quelconque liberté. Le sujet de l'écoute est mis en demeure d'exécuter purement et simplement la parole communiquée par l'ancien et véhiculée par la tradition ${ }^{25}$. Manifester une insoumission à cette parole est le signe d'un manque d'éducation et de respect à la tradition, voire à la personne de l'ancien. En conséquence, l'individu peut courir le risque d'être exclu de la communauté familiale, puisqu'il ne permet pas que le courant de la parole principielle délivrée par le premier ancêtre - parole dans et par laquelle la famille retrouve son identité et sa cohésion - puisse passer par lui. Ce qui est en jeu ici est l'aspect contraignant de l'écoute quant au rapport de l'individu à la communauté. De fait, la communauté attachée à la tradition prend tellement de l'importance qu'elle laisse peu de place à l'éclosion de la personne humaine capable d'initiative et d'auto-prise en charge.

25 Cf. J. M. H. Quenum, «De la sagesse des ancêtres à la Parole de Dieu», in RUCAO 24 (2005), p. 155. 
Par contre, dans la dynamique de l'écoute biblique, ce qui domine est la confiance manifestée au Dieu d'amour qui a tellement aimé le monde qu'il a donné son Fils unique (Parole de Dieu incarnée) afin que quiconque croit en lui, dans l'acte d'obéissance-confiante, ne périsse pas mais ait la vie éternelle (cf. Jn 3,16). C'est en effet en toute liberté et du fait de l'amour manifesté par le Dieu d'amour que le sujet de l'écoute s'engage volontairement, au risque de sa vie, à mettre en pratique la Parole de Dieu. Autrement dit, l'écoute-obéissante ici «n'évoque donc pas une idée de contrainte, de soumission aveugle, mais une libre adhésion du cœur, un acte de foi et d'amour ${ }^{26}$.

Par conséquent, l'ouverture au Dieu d'amour, du fait de l'acte libre et volontaire qui la sous-tend, inscrit le sujet de l'écoute dans un rapport de type kénotique et extatique, en ce sens que, se décentrant de lui-même (rapport kénotique), ce dernier s'ouvre à Dieu et au prochain (rapport extatique) pour aimer à son tour de tout son cœur, de tout son être et de toute sa force ( Dt, 6, 5; Lc 10,27). C'est dans ce rapport de foi et d'amour que la parole écoutée peut être fondatrice des rapports de fraternité aux dimensions universelles dans la nouvelle famille de Dieu en Afrique.

26 M. Hubaut, Op. Cit., p. 119. 


\section{Références}

Adoukonou, B., Jalons pour une théologie africaine. Essai d'une herméneutique chrétienne du Vodoun dahoméen, T2, Etude ethnologique, Lethielleux, Paris, 1980.

Agossou, M.J., «La parole dans son mystère et sa révélation: $\mathrm{O}$ xo ma nyi mêdé gbê», in Eglise de Cotonou, N 8, août 1998, pp. 55-63.

Agossou, M. J., Christianisme africain. Une fraternité au-delà de l'ethnie, Paris, Karthala, 1987.

Calame-Griaule, G., Ethnologie et langage. La parole chez les Dogons., Paris, Gallimard, 1965.

Carriere, J.-M., Le livre du Deutéronome. Choisir la vie., Paris, Editions Ouvrières, 2002.

Chretien, J.-L., Saint Augustin et les actes de parole., Paris, PUF, 2002.

Christensen, D. L., World Biblical Commentary Vol. 6A. Deuteronomy I-II, Dallas, Word Books, Publisher, 1991.

Congar, Y., «'Ecouter' et 'voir' » in La vie Spirituelle., 1969, n 560, pp. 501-511.

Cousin, H., L'Evangile de Luc. Commentaire pastoral., Paris, Centurion, 1993.

Gregoire de Nysse, Homélie sur le Cantique des Cantiques., Paris, Migne, 1992.

Grelot, P., La Bible, Parole de Dieu. Introduction à l'étude de l'Ecriture Sainte, Coll. Bibl. de théologie, Série 1/5, Paris - Tournai, 1965.

Guedou, G., Xo et Gbe. Langage et Culture chez les Fon (Dahomey), 3 T, Thèse de Doctorat de 3ème cycle en linguistique, Paris, 1976.

Guillet, J., «Parole de Dieu », in Dictionnaire de Spiritualité, T. XII, Beauchesne, Paris, 1984.

Guillaume de Saint-Thierry, "Monition sur la méditation de la Parole de Dieu (Sermon 6-9)", in Sources Chrétiennes 175, Paris, Cerf, 1971.

Houngbedji, R., L'Eglise-famille de Dieu en Afrique selon Luc 8, 19-21. Problèmes de fondements, Paris, L'Harmattan, 2009.

Hubaut, M., Un Dieu qui parle! Comment Dieu se révèle-t-il à l'homme?, Paris, Cerf, 2012.

Quenum, J.-M. H., «De la sagesse des ancêtres à la Parole de Dieu», in RUCAO 24 (2005), p. 153-165.

Saint augustin, Les plus beaux sermons de saint Augustin. Réunis et traduits par le chanoine G. Humeau, Paris, Bayar, 1932.

Saint Cesaire D'Arles, Sermons au peuple, Tome I (Sermons 1-20), Paris, Cerf, 1971.

Soede, N.Y., «Parole et vérité dans la culture et les comportements actuels des africains», in RUCAO 24 (2005), p. 133-152. 